\title{
Urinary Incontinence: Revisited
}

\author{
${ }^{1}$ Narendra Malhotra, ${ }^{2}$ Ruchika Garg, ${ }^{3}$ Prabhat Agrawal, ${ }^{4}$ Rajani Rawat
}

\section{ABSTRACT}

Patients usually under-report symptoms of incontinence. Therefore, improved physician-patient communication is vital. Most urinary incontinence can be evaluated and treated after careful history and simple clinical assessment. Initial treatment, for both urge urinary incontinence and stress urinary incontinence, is lifestyle modification and pelvic floor muscle exercises.

Urgency responds to bladder training and drug therapy with anticholinergic medication. Pharmacotherapy has a limited place in stress incontinence.

Keywords: Urinary incontinence, Overactive bladder, Anticholinergic drugs.

How to cite this article: Malhotra N, Garg R, Agrawal P, Rawat R. Urinary Incontinence: Revisited. J South Asian Feder Obst Gynae 2014;6(2):107-111.

\section{Source of support: Nil}

Conflict of interest: None declared

\section{INTRODUCTION}

Urinary incontinence (UI) affects women of all ages, with a wide range of severity. Urinary incontinence may seriously influence the physical, psychological and social wellbeing of affected individuals. The field of female urology is evolving in India, but still much research is required.

\section{PATHOPHYSIOLOGY}

Continence is achieved by normal anatomical and physiological function of bladder, urethra, sphincter and also the nerves supplying these organs. A defect in any of these can cause incontinence.

\footnotetext{
${ }^{1,3}$ Consultant, ${ }^{2,4}$ Assistant Professor

${ }^{1}$ Department of Obstetrics and Gynecology, Rainbow Hospital and IVF Center, Agra, Uttar Pradesh, India

${ }^{2}$ Department of Obstetrics and Gynecology, SN Medical College, Agra, Uttar Pradesh, India

${ }^{3}$ Department of Medicine, SN Medical College, Agra, Uttar Pradesh, India

${ }^{4}$ Department of Obstetrics and Gynecology, Rural Institute of Medical Sciences, Itawah, Uttar Pradesh, India

Corresponding Author: Ruchika Garg, Assistant Professor Department of Obstetrics and Gynecology, SN Medical College, Agra, Uttar Pradesh, India, e-mail: ruchikagargsnmc@ gmail.com
}

At the initial clinical visit, the woman's urinary incontinence should be categorized as stress UI (SUI), mixed UI (when symptoms of stresss and urge incontinence occur together, or urge UI/overactive bladder syndrome (OAB). Uninary incontinence has an estimated prevalence of $15 \%$ in ambulatory adult population. ${ }^{1}$ SUI is the most common form of UI (Hampel et al).

Urgency may be motor urgency due to bladder overactivity or sensory urge due to hypersensitivity.

Urethral sphincter incompetence leds to Stress incontinence. Urethral smooth muscle, external urethral sphincter, inner urethral factor, pressure transmission to bladder and urethra, pelvic floor musculature, hormones, connective tissue, and nerves are all involved in the maintenance of urethral competence.

Overactive bladder (OAB) syndrome is a common condition characterized by urgency with or without urge incontinence usually with frequency and nocturia. The prevalence of overactive bladder in women aged 40 years and above is $17.4 \%$ and the prevalence increases with age in both sexes. The symptoms of urgency and or frequency are equally common in both sexes but urge incontinence is more common in women (Milsom 2001).

Overflow incontinence means involuntary loss of urine associated with overdistension of the bladder due to inefficient bladder emptying. Poor detrusor contractility or bladder outlet obstruction or a combination of both can lead to it. Mechanical obstruction or functional disorder can also be the culprits.

\section{CONTINUOUS INCONTINENCE}

It results from a fistula among the ureter, bladder or urethra, and the vagina, or an ectopic ureter opening into the vagina or urethra.

Reflex incontinence is due to detrusor hyperreflexia and/ or involuntary urethral relaxation in the absence of sensation. It is not usually associated with the desire to micturate. This is highly suggestive of a neurological cause.

Increasing age, childbirth, obesity, presence of lower urinary tract symptoms and decreased mobility (Hunskaar 2002) are associated with UI.

\section{ASSESSMENT AND INVESTIGATIONS}

International consultation on incontinence modular questionaire urinary incontinence (ICIQ-UI) is a simple screening 
tool for quality of life assessment consisting of four questions; how frequently the incontinence occurs, the volume leaked, impact on quality of life and the type of incontinence. It is to be recommended for use in outpatient clinics. A history of sudden-onset urge urinary incontinence may be the first sign of an underlying bladder tumor in a older women.

A bimanual examination should be done to exclude pelvic masses and pelvic organ prolapse. Prolapse should be assessed objectively, using the International Continence Society pelvic organ prolapse quantification system (POPQ). Pelvic floor assessment should be done clinically.

\section{Urine Testing}

A urine dipstick test is a must in all women for presence of blood, glucose, protein, leukocytes and nitrites in the urine.

Postvoid residual volume should be measured by ultrasonography in patients with symptoms suggestive of voiding dysfunction or recurrent urinary tract infection.

\section{Pad Test}

Pad tests are not recommended in the routine assessment of women with UI. In some cases, where incontinence cannot be demonstrated phenazopyridine hydrochloride $\left(\right.$ Pyridium $^{\circledR}$ ) $200 \mathrm{mg}$ three times daily for 48 hours will stain the urine orange and any leakage can be identified on a pad.

One-hour pad test is a semiobjective measure to help identify the volume lost. A 24-hour pad test is a better assessment.

\section{When to consult Urology Specialist?}

If microscopic or visible hematuria in women aged 50 years or recurrent or persistent urinary tract infection associated with hematuria, or any suspected malignant mass of urinary tract is present consultation from urology specialist should be taken. If bladder is palpable on pelvic examination after voiding, patient should be referred to a urogynecologist. ${ }^{2}$

Bladder diaries should be used in the initial assessment of women with UI or OAB. A minimum of 3 days of the diary should be maintained taking into account both working days and holidays. Bladder diaries are a reliable method of quantifying urinary frequency and incontinence episodes. With a 3-day period compliance can be assured.

\section{Grading of Recommendations}

\begin{tabular}{ll}
\hline Grade & Recommendation \\
\hline A & $\begin{array}{l}\text { Requires at least one randomized controlled trial as } \\
\text { part of the body of literature of overall good quality and } \\
\text { consistency. }\end{array}$ \\
B $\quad \begin{array}{l}\text { Requires well-conducted clinical studies but no } \\
\text { randomized clinical trials. }\end{array}$ \\
C $\quad \begin{array}{l}\text { Requires evidence from expert committee reports } \\
\text { or opinions and/or clinical experience of respected } \\
\text { authorities. Indicates absence of directly applicable } \\
\text { studies of good quality. }\end{array}$ \\
\hline
\end{tabular}

\section{Recommendations for Clinical Practitioners}

\section{Role of Urodynamics}

Multichannel cystometry or urodynamics are not recommended before starting conservative treatment.

It is indicated before surgery ${ }^{2}$ for UI if:

- Detrusor overactivity is to be excluded or

- It is a case of failed surgery for stress incontinence or anterior compartment prolapse, or

- Symptoms of voiding dysfunction are present (Grade B). Ambulatory urodynamics or videourodynamics may also be considered in these circumstances. Urodynamics must be performed with the emphasis on reproducing patient's symptoms. Staff should be fully trained to recognize and correct artefacts, and to interpret tracings (Grade C).

- If it is necessary to assess urethral function, either urethral closure pressures or abdominal leak-point pressures are appropriate (Grade C).

- Urine flow rate and post-void residual measurement are useful screening tests which indicate voiding dysfunction: only pressure-flow studies of voiding can define the cause of voiding dysfunction (Grade C). ${ }^{2}$

- Bladder wall thickness may be useful for the detection of detrusor overactivity as long as bladder outlet obstruction is excluded with uroflowmetry and postvoid residual estimation (Grade C).

- Investigations of choice in suspected fistulae are examination under anesthesia, cystourethroscopy and dye testing (Grade $\mathrm{C}$ ).

- Urodynamic investigations will rarely alter the approach to management of urinary fistulae but may influence counseling (Grade C).

\section{TREATMENT}

\section{Role of Pelvic Floor Muscle Training}

Daily pelvic floor muscle training continued for 3 months is a safe and effective first-line treatment for stress and mixed UI.

Bladder training for a minimum of 6 weeks should be offered as first-line treatment to women with urge or mixed UI. A time table for voiding is given and the patient has to void at a scheduled interval. What is the mechanism of action behind bladder training is uncertain. One hypothesis is that as the interval between voids increases, the bladder capacity increases, leading to a reduction in urinary incontinence (Wilson 2002).

Women should be motivated to perform pelvic-floor muscle exercises intensively as the first line of treatment. More than 30\% do not contract correctly at their first consultation and thorough individual attention is needed. Manual techniques and electrical stimulation may be used to teach how to contract. ${ }^{2}$ 
Urgency-frequency syndrome is a diagnosis of exclusion of both organic and nonorganic pathology. Endometriosis and genital atrophy can also produce lower urinary tract symptoms.

The international consultation on incontinence recommends anticholinergics as first line pharmacotherapy in urge incontinence for men and women, the elderly and patients with detrusor hyper-reflexia. Before other treatment options are considered urodynamic studies should be done.

Estrogens cannot be used to treat stress incontinence. (Grade C).

Estrogen therapy, especially vaginal estrogens in postmenopausal women can improve urgency, frequency and recurrent urinary tract infections (Grade C). ${ }^{3}$

Surgical therapy may also be considered in older women and a multidisciplinary approach should be practiced (Grade C).

\section{Overactive Bladder}

The pathophysiology of the overactive bladder remains to be fully elucidated. The involvement of the autonomic nervous system in bladder/detrusor function is recognized (de Groat). The motor supply to the bladder is via the parasympathetic sacral nerves S2, 3, 4 which effects detrusor muscle contraction. This is mediated by acetylcholine acting on muscarinic receptors at the level of the bladder. ${ }^{4}$

The rationale for using anticholinergic drugs in the treatment of overactive bladder syndrome is to block the parasympathetic acetylcholine pathway and thus reduce the intensity of detrusor muscle contraction.

The two main treatment options for overactive bladder are pharmacotherapy and conservative management (e.g. bladder training or electrical stimulation) or a combination of both. Other drugs have both peripheral and central action (Andersson 2000).

1. Tricyclic antidepressants and monoamine reuptake inhibitors: For example imipramine, amitryptyline and duloxetine. Imipramine is successfully used in the treatment of nocturnal enuresis in children. (Hunsballe 2001), In the elderly as well it has also found to be useful (Castleden 1986). At present it is not in widespread clinical use for overactive bladder.

2. Afferent nerve inhibitors: For example, lidocaine, dimethyl sulphoxide DMSO, capsaicin which all act as local anesthetics and vanilloid receptor agonists, thereby reducing afferent input which might otherwise trigger micturition. None of these drugs are currently in widespread use. Capsaicin has been administered intravesically with success in neurogenic bladder patients but the effect may only last for several months (2nd ICI 2001).
3. Botulinum-A toxin: This drug selectively blocks the parasympathetic nerve transmission to the detrusor muscle at the local bladder level. The use of intravesical botulinum toxin is increasing especially in resistant cases of neurogenic and idiopathic overactive bladder with encouraging results (Dmochowski 2007).

4. Alpha-adrenergic antagonists: For example, tamsulosin, alfuzosin and doxazosin which act by reducing bladder outlet resistance but may also act centrally to inhibit micturition. Their role in overactive bladder is unclear at present.

5. Flavoxate: The main mechanism of action is not yet fully established.

It is a calcium channel blocker, a local anesthetic, as well a phosphodiesterase blocker. No anticholinergic activity has been demonstrated. This drug is no longer in widespread clinical use in overactive bladder and two randomized controlled trials have demonstrated no benefit vs placebo (Chapple 1990; Dahm 1995).

Four trials (Gaudenz 1978; Meyhoff 1981; Riva 1989; Wehnert 1989) reported that there were no statistically significant differences between flavoxate and anticholinergic drugs. Gaudenz 1978 found more patients preferred flavoxate although objective assessment with urodynamics was equivocal. Milani 1993 et al found flavoxate was the preferred drug. Two crossover trials (Cardozo 1979; Milani 1993) reported favorable results for anticholinergics.

Results of review of nine studies comparing flavoxate and anticholinergics favored the use of anticholinergics in preference to flavoxate for overactive bladder. Side effect were more in the anticholinergic groups. But the newer anticholinergics have less side effects. There is no evidence to suggest flavoxate should be used in treatment of overactive bladder. ${ }^{5}$

6. Others: Includes calcium channel blockers like verapamil, potassium channel openers like pinacidil and cromakalim and Gamma-aminobutyric acid GABA agonists like baclofen.

Verapamil has been reported to increase bladder capacity in detrusor hyper-reflexia when instilled intravesically (Mattiasson A), but the drug is not in widespread clinical use at present. Verapamil had no significant effect $v s$ placebo in a study.

Oral nifedipine has been used as prophylaxis for autonomic hyperreflexia during bladder instrumentation with success (Wein 2001). Potassium channel blockers such as cromakalim and pinacidil have been effective in animal models but have not been studied in human overactive bladder.

Baclofen has been described in detrusor hyperreflexia but other published evidence is lacking. These drugs are not in current clinical use in overactive bladder (2nd ICI 2001). 


\section{What is the Place of Anticholinergic Drugs?}

Anticholinergic drugs are better than alpha adrenergic antagonists. Serels 1998 studied hyoscyamine vs doxazosin. Mean improvements was similar in both groups: A higher incidence of adverse effects was seen in the anticholinergic group. No statistically significant differences in urodynamic parameters were reported. The study suggests those who failed to respond to one medication responded to the other ( $50 \%$ of women not responding to hyoscyamine responded to doxazosin whilst $38 \%$ of women not responding to doxazosin improved with hyoscyamine).

In a study comparing lidocaine given via bladder catheter and oral emepronium found significantly better urodynamic results in the lidocaine group (Ednzelsberger 1991). Afferent nerve inhibitors diminish in effectiveness after several months treatment (e.g. capsaicin) (2nd ICI 2001). Long-term effects of lidocaine are not known. This is an area requiring further research to fully establish the role of afferent nerve inhibitors in overactive bladder.

Intravesical oxybutynin was significantly better than verapamil in one-off doses in a single trial (Frohlich 1998). There were no demonstrable improvements in urodynamic parameters with verapamil. ${ }^{5}$

1. Intravesical botulinum toxin ${ }^{6}$ appears to be an effective therapy for refractory OAB symptoms, but as yet little data exist on benefits and safety compared with other interventions, or with placebo. Further data are required on long-term outcomes, safety, and optimal dose of botulinum toxin for OAB.

2. Lower doses of botulinum toxin (100 to $150 \mathrm{U}$ ) have beneficial effects, but larger doses (300 U) may be more effective and longer lasting, but with more side effects.

3. Suburothelial injection had comparable efficacy to intradetrusor injection. The effect of botulinum toxin may last for a number of months and is dependent upon dose and type of toxin used. Repeated doses do not lead to refractoriness to botulinum toxin. Botulinum toxin appeared to have beneficial effects in $\mathrm{OAB}$ that quantitatively exceeded the effects of intravesical.

The United States food and drug administration's (FDA) manufacturer and user facility device experience (MAUDE) database has recorded self-reported incidences of major complications with the use of TVT needle. They are not specific to the TVT needle, but are operator-dependent. Vascular, bowel, bladder and urethral injury can occur with all these devices. The proper use of the instruments should be learned.

\section{What are the Recommendations in Pregnancy?}

1. Every antenatal patient should be asked about urinary and fecal continence. Pregnancy per se and delivery appear to be equally responsible for the risk of urinary symptoms; increasing parity increases these risks. Before atttempting the next pregnancy the effects of pregnancy and mode of delivery, should be discussed with the patients. Antenatal pelvic floor muscle exercises reduces the incidence of postnatal UI.

2. Episiotomy does not appear to protect the pelvic floor or prevent stress incontinence (Grade B).

3. Cesarean section is not completely protective against urinary and fecal incontinence (Grade B).

4. Postnatal pelvic-floor exercises are effective in reducing urinary incontinence (Grade A).

5. Antenatal pelvic-floor exercises are effective in risk groups (Grade A).

6. Treat complex gastrointestinal disease during pregnancy (Grade C).

\section{What are the Recommendations for Surgical Practise}

\section{Sub-urethral Sling}

According to cochrane review minimally invasive synthetic suburethral sling ${ }^{7}$ operations appeared to be as effective as open retropubic colposuspension with fewer perioperative complications, less postoperative voiding dysfunction, shorter operative time and hospital stay but significantly more bladder perforations.

There was conflicting evidence about the effectiveness of suburethral slings compared to laparoscopic colposuspension in the short-term. Suburethral slings had significantly less de novo urgency and urgency incontinence, shorter operating time, hospital stay and time to return to daily activities.

A retropubic bottom-to-top route was more effective than top-to-bottom route and incurred significantly less voiding dysfunction, bladder perforations and tape erosions.

Monofilament tapes had significantly higher objective cure rates compared to multifilament tapes and fewer tape erosions.

The obturator route was less favorable than the retropubic route in objective cure, although there was no difference in subjective cure rates. However, less voiding dysfunction, blood loss, bladder perforation and shorter operating time has been reported with the obturator route.

Laproscopic colposuspension ${ }^{8}$ is as good as open colposuspension 2 years postsurgery. Vaginal slings have better objective outcomes in the short-term and similar subjective outcomes in the long-term.

In laproscopic colposuspension use of two paravaginal sutures is the most effective measure. 


\section{CONCLUSION}

Key to evaluation and management of UI is directly inquiring about it because about $30 \%$ of patients willnot spontaneously report it.

Significant clinical data on UI has been generated during the last 20 years. Since, the patients are hesitant to disclose the problem of incontinence leading questions regarding incontinence should be a routine part of history taking in gynecology. History alone is a very efficient tool to reach at a diagnosis. Thorough history, with a questionnaire, voiding diary, some measure of quantifying urinary loss, quality of life assessment, urine analysis and postvoid residual (PVR) measurement is adequate for a pure stress urinary incontinence or initial management of $\mathrm{OAB}$ symptoms. Additional workup may be required in patients with failed prior surgery, mixed incontinence, unexplained large PVR and those with neuropathic history.

\section{REFERENCES}

1. Berghmans L, Hendriks H, De Bie R, Van Waalwijk E, Van Doorn Bø K, Van Kerrebroeck P. Conservative treatment of urge urinary incontinence in women: a systematic review of randomized clinical trials. BJU International 2000;85:254-263.

2. Nice clinical guideline 40 . Urinary incontinence: the management of urinary incontinence in women. 2006 Oct.

3. MacLean AB, Cardozo L. Recommendations arising from the 42nd Study Group: Incontinence in Women. London: Royal College of Obstetricians and Gynaecologists Press 2002; p. 433-441.

4. Nabi G, Cody JD, Ellis G, Hay-Smith J, Herbison GP. Anticholinergic drugs versus placebo for overactive bladder syndrome in Cochrane Incontinence Group Published Online: 21 Jan 2009 DOI: 10.1002/14651858.CD003781.pub2

5. Roxberg C, Cook J, Dublin N. Anticholinergic Drugs versus other medications for overactive bladder in adults Cochrane Review Issue 3, JUN 2007 DOI: 10.1002/14651858.CD003190. pub4.

6. Duthie JB, Vincent M, Herbison GP, Wilson DI, Wilson D. Botulinum toxin injections for adults with overactive bladder syndrome, Cochrane Incontinence Group. Feb 2010 DOI: 10.1002/14651858.CD005493.pub3.

7. Ogah J, Cody JD. Lynne Rogerson Minimally invasive synthetic suburethral sling operations for stress urinary incontinence in women, Cochrane Incontinence Group, June 2009 DOI: 10.1002/14651858.CD006375.pub2

8. Dean N, Ellis G, Herbison GP, Wilson D. Laparoscopic Colposuspension for urinary incontinence in women, Cochrane Databases of Systemic Reviews 2010(2), Art no.:CD002239. DOI.10.1002/14651858. 